\title{
Article
}

\section{An Association between Lower Extremity Function and Cognitive Frailty: A Sample Population from the KFACS Study}

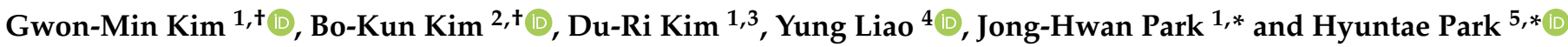 \\ 1 Health Convergence Medicine Laboratory, Biomedical Research Institute, Pusan National University \\ Hospital, Busan 49241, Korea; rlarnjsals47@gmail.com (G.-M.K.); drkim4100@gmail.com (D.-R.K.) \\ 2 Faculty of Sports Health Care, In-je University, Gimhae 50834, Korea; fabulousbobo79@gmail.com \\ 3 Department of Occupational Therapy, Graduate School of Inje University, Gimhae 50834, Korea \\ 4 Department of Health Promotion and Health Education, National Taiwan Normal University, \\ Taipei 106, Taiwan; liaoyung@ntnu.edu.tw \\ 5 Department of Health Sciences, Graduate School, Dong-A University, Busan 49315, Korea \\ * Correspondence: parkj@pusan.ac.kr (J.-H.P.); htpark@dau.ac.kr (H.P.); Tel.: +82-51-240-7071 (J.-H.P.); \\ +82-51-200-7979 (H.P.) \\ + These authors contributed equally to this work.
}

check for updates

Citation: Kim, G.-M.; Kim, B.-K.; Kim, D.-R.; Liao, Y.; Park, J.-H.; Park, H. An Association between Lower Extremity Function and Cognitive Frailty: A Sample Population from the KFACS Study. Int. J. Environ. Res. Public Health 2021, 18, 1007. https:// doi.org/10.3390/ijerph18031007

Academic Editors: Byoung-Hee Lee, Song-Young Park, Yi-Sub Kwak and Elizabeth Pekas

Received: 25 September 2020

Accepted: 19 January 2021

Published: 23 January 2021

Publisher's Note: MDPI stays neutral with regard to jurisdictional claims in published maps and institutional affiliations.

Copyright: (c) 2021 by the authors. Licensee MDPI, Basel, Switzerland This article is an open access article distributed under the terms and conditions of the Creative Commons Attribution (CC BY) license (https:// creativecommons.org/licenses/by/ $4.0 /)$.

\begin{abstract}
The present study aimed to define the physical function and lipid profile for physical and cognitive frailty in community-dwelling Korean older adults. A total of 229 participants (age $=76.76 \pm 3.72$ years; body mass index $=24.83 \pm 3.15$ ) were classified into four groups: robust, pre-frailty, cognitive decline, and cognitive frailty. An analysis on the four groups was performed to measure their physical and cognitive function, as well as blood biomarkers. The area under (AUC) the receiver operating characteristic curve (ROC) indicated that the 6-min Walk Test (6MWT), Timed Up and Go test (TUG), and Five Times Sit-to-Stand test (FTSS) had the potential to distinguish the capacity of an old adult to predict cognitive frailty. The 6MWT had a higher sensitivity and the TUG and FTSS tests had greater specificity. With cognitive frailty as a categorical variable, cognitive frailty status was a significant predictor of the TUG (odds ratio (OR) 2.897; 95\% confidence interval (CI), 1.283-6.541), FTSS (OR 3.337; 95\% CI 1.451-7.673), and 6MWT (OR 0.204; 95\% CI 0.070-0.591) tests. Our findings indicate that the 6MWT, TUG, and FTSS tests are closely related to cognitive frailty and can provide potential prognostic cutoff points.
\end{abstract}

Keywords: cognitive frailty; Timed Up and Go test; Five Times Sit-to-Stand test; 6-min Walk Test; lower extremity function

\section{Introduction}

Frailty is associated with chronic diseases and poor prognoses, such as death or disability [1-4]. Among the multiple dimensions of frailty, physical frailty has been widely recognized as a public health priority in older adults, while the term "cognitive frailty" has been coined in the literature in recent years [5]. An international consensus group has proposed the concept of cognitive frailty as a heterogeneous cognitive state characterized by the simultaneous presence of both cognitive impairment and physical frailty, excluding concurrent or other dementias [6]. Recent research has shown that the relationship between cognitive decline and physical frailty has become more overt $[7,8]$. Furthermore, previous studies reported that cognitive frailty was related to a higher prevalence of obesity, low physical function, and disability rather than to physical frailty [9-11].

Regarding the relationship between physical and cognitive frailty, there is a reduction in physical function through cognitive decline, and this reduction in physical capacity reduces the functionality of old adults to perform complex motor tasks. This is related to a reduction in functional and impaired mobility [12]. Cognitive decline, in conjunction with declining physical function, has been related to an increased risk for dementia and 
disability in population-based research of older adults [13,14]. In particular, lower extremity weakness increases with age [15], resulting in substantial differences in lower extremity function between young and older adults [16-18]. Lower extremity weakness in older adults is also an independent predictor of future falls [19,20]. In addition, there is growing evidence that declined lower extremity function is also present in milder phases, which may be related to the level of cognitive decline [13,21].

However, a previous systematic review indicated that the findings of a relationship between changes in cognitive and physical functions are relatively small, owing to a few studies reporting an association [22]. In addition, it is unclear whether lower extremity weakness is associated with cognitive frailty in Korean older adults. Therefore, the present study aimed to compare the performance of different lower extremity function tests in wellcharacterized individuals with or without cognitive frailty, along with controls matched demographically and according to comorbidities, such as obesity and depression.

\section{Materials and Methods}

This study was approved by the Dong-A University Research Ethics Committee (IRB number is BR-009-019). All subjects provided their informed written consent prior to participation, which was obtained from all participants after they were provided with a detailed description of the experiment, in accordance with the ethical standards of the Declaration of Helsinki.

\subsection{Participants}

A total of 251 Korean older adults were recruited through an advertisement placed in the local health center and newspapers at Pusan, Korea. All applicants had to meet the following criteria to be included in the study: (1) age greater than 65 years; and (2) not diagnosed with a terminal illness. As shown in Figure 1, of the 251 initial participants, we excluded 19 based on the inclusion criteria. We then excluded three more applicants who were not eligible for the present study because they were illiterate, had a hip or knee joint arthroplasty within the preceding 6 months, or were unable to walk independently. In the end, 229 participants were included in the present study.

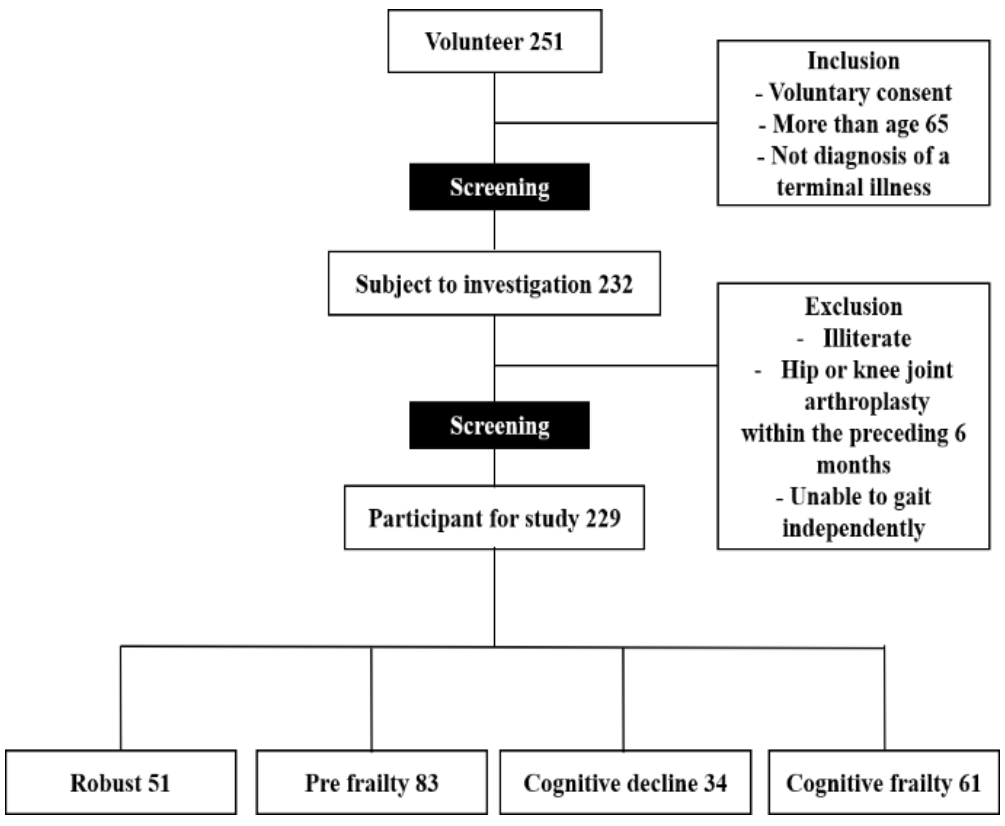

Figure 1. Study flow diagram. 


\subsection{Cognitive Function}

The Mini-Mental State Examination-Dementia Screening (MMSE-DS) [23] was used to screen for cognitive decline. When the score was $<25$, this was defined as cognitive decline. This test is one of the best known and most affordable cognitive screening instruments available for research and clinical practice [17].

\subsection{Defined Cognitive Frailty}

The five components of a physical frailty phenotype, which were assessed according to a pre-defined protocol, are self-reported exhaustion, unintentional weight loss, reduced grip strength, reduced gait speed, and self-reported low physical activity [18]. Depending on the number of criteria attained, individuals were classified as robust (0), pre-frail (1-2), and frail (3-5). However, in the present study, participants were divided into four groups as follows: (A) robust (physical frailty score of 0; MMSE score $>25$ points); (B) pre-frailty (physical frailty score $>1$; MMSE score $<25$ points); (C) cognitive decline (physical frailty score of 0; MMSE score < 25 points); and (D) cognitive frailty (physical frailty score $>1$; MMSE score $<25$ points) [9].

\subsection{Body Composition}

The body mass index and appendicular skeletal muscle mass index of all participants were measured using a bioelectrical impedance analysis. Body composition values were determined using an Inbody S10 device (S10; Inbody, Seoul, Korea, 2014). Waist circumference was measured at the narrowest part between the lower rib and the iliac crest (the natural waist) or, in case of an indeterminable waist narrowing, halfway between the lower rib and the iliac crest. On the other hand, hip circumference was measured over the widest part of the buttocks. Both measurements were recorded to the nearest half centimeter. The waist-to-hip ratio was calculated as the waist measurement divided by the hip measurement.

\subsection{Blood Pressure and Blood Test}

Arterial blood pressure was measured from the right arm in a seated position using a blood pressure monitor (HEM-7121; Omron Corporation, Kyoto, Japan, 2016). A blood analysis was performed on the plasma samples $(5 \mathrm{~mL})$ of the participants. A blood sample was drawn from an antecubital vein of participants between 08:00 and 10:00 a.m., after a 12-14 h overnight fast. The samples were collected in a seated position according to a standard protocol, and they were centrifuged within 30-45 min upon collection. Analysis of the samples was performed using auto-analyzers (Hitachi 902; Roche, Manheim, Germany, 1997). The samples were then collected into tubes containing clotting activators to isolate the serum. Triglyceride, total cholesterol, high-density lipoprotein cholesterol (HDL-C), low-density lipoprotein cholesterol, glucose, insulin, and creatinine kinase were analyzed using standard lipid profiles for older adults.

\subsection{Physical Function}

The hand grip strength of the dominant hand of participants was measured with a handheld dynamometer (TKK 5401; Takei Scientific Instruments, Tokyo, Japan, 2012). They were instructed to perform a maximal isometric contraction. During the assessment, the participants were asked to stand upright with their feet shoulder-width apart and to look forward with the elbow fully extended. The test was repeated after $30 \mathrm{~s}$, and the highest value was recorded. The Five Times Sit-to-Stand test (FTSS) was then used to assess leg strength and endurance. Participants were instructed to cross their arms across the chest and stand up and sit down five times, as fast as possible, with the time measured in seconds [19]. For the Timed Up and Go test (TUG), related to leg function, a 3-m walkway was marked on a flat surface using a cone on the floor; the chair used had back support but no armrests (seat height: $46 \mathrm{~cm}$ ). Participants were then asked to stand up from the chair, walk forward for the 3-m distance as rapidly as possible, walk back, and return to sit 
on the chair. Gait speed was measured in the standard gait task protocol, with the time measured in seconds. The gait speed test used a 7-m distance, which included a 1.5-m acceleration and deceleration distance; only the 4-m walk was timed for gait speed. Lastly, the 6-min Walk Test (6MWT) was used to examine the aerobic capacity of the participants since the test is specifically tailored and self-paced for older adults [20]. Each participant was instructed to walk as far as possible accompanied, to avoid any interference in walking speed, along a $20 \mathrm{~m}$ flat, obstruction-free corridor with one chair on both ends of this corridor. Participants were then instructed to walk around the cones at a fast speed for $6 \mathrm{~min}$; not to run or jog. The hand grip strength and gait speed are some of the essential variables to screen for frailty [18]. The validity and reliability of all of the tests that are employed in the present study were adequately proved via lots of previous studies that investigated the physical function of older adults [24-26].

\subsection{Statistical Analysis}

The statistical analysis was carried out using the IBM SPSS Statistics for Windows version 21.0. (IBM Corp: Armonk, NY, USA). The characteristics of the study population are presented as the mean \pm standard deviation (SD) for continuous variables and as proportions for categorical variables. One-way analysis of variance (ANOVA) was carried out to compare the anthropometric measures, physical function, and biomarker differences between the four groups. Post-hoc Bonferroni tests were performed after one-way ANOVA to look for differences between the groups, physical functions, and blood biomarkers. Subsequently, we used logistic regression analyses to determine the odds ratio and $95 \%$ confidence intervals, adjusted for sex and age, and to assess the independent associations between physical function and the risk of cognitive frailty. Statistical significance was defined as $p<0.05$. The best cutoffs for predicting physical function to cognitive frailty were evaluated by the parameters (6MWT, TUG, and FTSS) provided by the receiver operating characteristic curve (ROC), the area under the ROC curve (AUC), sensitivity, and specificity. Analyses were performed using MedCalc for Windows version 9.1.0.1 (MedCalc ${ }^{\circledR}$ Software Corporation, Mariakerke Ostend, Belgium, http:/ / www.medcalc.be).

\section{Results}

\subsection{Baseline Characteristics of the Participants}

The mean (SD) age of the participants was 76.7 (3.7) years, and $80.3 \%$ of participants were women. Table 1 shows the measures (mean and SD) for the physical, cognitive, functional, and blood biomarkers in the present study.

\subsection{Comparisons of Physical Function and Blood Biomarkers Using ANOVA Followed by Pairwise Comparisons Using the Bonferroni Test}

As a result of comparing the physical function of the participants in terms of the four groups, a significant difference was found in the 6MWT $(p<0.02)$, FTSS $(p<0.00)$, and TUG $(p<0.00)$ levels. A post-hoc test showed that the values of the 6MWT were significantly lower in group A4 (cognitive frailty) than in group A1 (robust); the FFSS values were significantly lower in group A3 (cognitive decline) and A4 compared to group A1; and that the TUG values were significantly lower in group A3 and A4 compared to group A1, while that of group A4 was significantly lower compared to group A2 (Table 2).

\subsection{For Multi-Nominal Logistic Regression Models Predicting Cognitive Frailty from Physical Function}

The results of the logistic regression analyses are shown in Table 3. With cognitive frailty as a categorical variable, TUG status was a significant predictor at $\geq 8.21$ (OR 2.790, 95\% CI 1.266-6.151; adjusted model: OR 2.897, 95\% CI 1.283-6.541); FTSS status was a significant predictor at $\geq 12.54$ (OR 3.115, 95\% CI 1.390-6.982; adjusted model: OR 3.337, $95 \%$ CI 1.451-7.673); and 6MWT status was a significant predictor at $\geq 420.01$ (OR 0.241 , 95\% CI 0.088-0.662; adjusted model: OR 0.204; 95\% CI, 0.070-0.591). 
Table 1. Baseline characteristics of the participants $(n=229)$.

\begin{tabular}{|c|c|}
\hline Basic Factor & \\
\hline Female (no, \%) & $184(80.3)$ \\
\hline Age (year) & $76.76 \pm 3.72$ \\
\hline Body mass index $\left(\mathrm{kg} / \mathrm{m}^{2}\right)$ & $24.83 \pm 3.15$ \\
\hline Appendicular skeletal muscle (kg) & $15.40 \pm 3.15$ \\
\hline Waist-hip ratio $(\%)$ & $0.93 \pm 0.07$ \\
\hline Systolic blood pressure (mmHg) & $138.81 \pm 18.00$ \\
\hline Diastolic blood pressure (mmHg) & $74.18 \pm 11.22$ \\
\hline \multicolumn{2}{|l|}{ Cognitive function } \\
\hline Mini mental state examination (point) & $24.74 \pm 3.54$ \\
\hline \multicolumn{2}{|l|}{ Physical function } \\
\hline 6 minute walk test $(\mathrm{m})$ & $378.11 \pm 63.25$ \\
\hline Five times sit to stand test (s) & $10.96 \pm 3.60$ \\
\hline Timed up and go test (s) & $7.33 \pm 1.52$ \\
\hline Grip strength $(\mathrm{kg})$ & $22.03 \pm 5.74$ \\
\hline Gait speed $(\mathrm{m} / \mathrm{s})$ & $1.14 \pm 0.18$ \\
\hline \multicolumn{2}{|l|}{ Bio-marker } \\
\hline Total cholesterol (mmol/L) & $4.76 \pm 4.76$ \\
\hline Triglycerides $(\mathrm{mmol} / \mathrm{L})$ & $1.45 \pm 0.64$ \\
\hline Low density lipoprotein cholesterol (mmol/L) & $3.07 \pm 0.91$ \\
\hline High density lipoprotein cholesterol (mmol/L) & $1.40 \pm 0.35$ \\
\hline Glucose $(\mathrm{mmol} / \mathrm{L})$ & $2.55 \pm 0.46$ \\
\hline Insulin $(\mathrm{mmol} / \mathrm{L})$ & $8.41 \pm 6.01$ \\
\hline Creatinine kinase ( $\mu$ kat/L) & $1.74 \pm 2.24$ \\
\hline
\end{tabular}

Data represent mean $\pm \mathrm{SD}$ and number, $\%$

Table 2. Comparison of physical function and blood biomarkers in old adults for 4 groups using a one way ANOVA followed by pair-ways comparison using a Bonferroni correction.

\begin{tabular}{|c|c|c|c|c|c|c|}
\hline Physical Function & A 1 (51) & A 2 (83) & A 3 (34) & A 4 (61) & $p$ & Post-Hoc \\
\hline 6 min walk test $(\mathrm{m})$ & $397.27 \pm 65.11$ & $381.77 \pm 74.94$ & $372.65 \pm 47.61$ & $360.16 \pm 45.52$ & 0.02 & A $1>$ A 4 \\
\hline Five times sit to stand test(s) & $9.69 \pm 2.41$ & $10.60 \pm 3.63$ & $11.84 \pm 3.79$ & $12.01 \pm 3.92$ & 0.00 & A $1<$ A 3, A 4 \\
\hline Timed up and go test (s) & $6.74 \pm 1.10$ & $7.10 \pm 1.37$ & $7.74 \pm 1.45$ & $7.92 \pm 1.80$ & 0.00 & $\begin{array}{c}\text { A } 1<\text { A } 3, \text { A } 4 ; \\
\text { A } 2<\text { A } 4\end{array}$ \\
\hline Grip strength (kg) & $22.70 \pm 4.59$ & $22.68 \pm 6.03$ & $20.97 \pm 4.96$ & $21.18 \pm 6.49$ & 0.24 & - \\
\hline Gait speed (m/s) & $0.87 \pm 0.12$ & $0.92 \pm 0.19$ & $0.89 \pm 0.11$ & $0.91 \pm 0.13$ & 0.21 & - \\
\hline \multicolumn{7}{|l|}{ Biomarker } \\
\hline Total cholesterol (mmol/L) & $4.77 \pm 0.99$ & $4.75 \pm 0.94$ & $4.93 \pm 1.00$ & $4.68 \pm 0.86$ & 0.63 & - \\
\hline Triglycerides $(\mathrm{mmol} / \mathrm{L})$ & $1.37 \pm 0.59$ & $1.48 \pm 63.90$ & $1.54 \pm 0.62$ & $1.43 \pm 0.56$ & 0.67 & - \\
\hline $\begin{array}{l}\text { Low density lipoprotein } \\
\text { cholesterol (mmol/L) }\end{array}$ & $3.07 \pm 0.95$ & $3.05 \pm 0.93$ & $3.23 \pm 0.98$ & $3.01 \pm 0.83$ & 0.75 & - \\
\hline $\begin{array}{l}\text { High density lipoprotein } \\
\text { cholesterol (mmol/L) }\end{array}$ & $1.44 \pm 0.33$ & $1.39 \pm 0.36$ & $1.37 \pm 0.38$ & $1.38 \pm 0.36$ & 0.77 & - \\
\hline Glucose $(\mathrm{mmol} / \mathrm{L})$ & $5.33 \pm 0.90$ & $5.62 \pm 1.10$ & $5.60 \pm 0.86$ & $5.32 \pm 0.92$ & 0.18 & - \\
\hline Insulin $(\mathrm{mmol} / \mathrm{L})$ & $8.10 \pm 5.03$ & $7.61 \pm 5.47$ & $10.56 \pm 8.02$ & $8.56 \pm 6.04$ & 0.11 & - \\
\hline Creatinine kinase ( $\mu$ kat/L) & $2.23 \pm 3.11$ & $1.84 \pm 2.67$ & $1.55 \pm 0.96$ & $1.31 \pm 0.62$ & 0.16 & - \\
\hline
\end{tabular}

A $1=$ Robust; A 2 = pre frail; A $3=$ Cognitive decline; A 4 = Cognitive frail; One-way analysis of variance was used with a post hoc Bonferroni correction for multiple comparisons; Data represent mean \pm SD. $p$ valued less than 0.05 . 
Table 3. Four multi-nominal logistic regression models predicting physical function in cognitive frailty.

\begin{tabular}{ccc}
\hline & Robust Model & Adjust Model \\
\hline Timed up and go & odds ratio (CI 95\%) & odds ratio (CI 95\%) \\
\hline$<6.31$ & Ref. & Ref. \\
$6.31-7.10$ & $0.748(0.295-1.897)$ & $0.772(0.302-1.972)$ \\
$7.11-8.20$ & $1.080(0.458-2.546)$ & $1.120(0.464-2.703)$ \\
$\geq 8.21$ & $2.790(1.266-6.151)$ & $2.897(1.283-6.541)$ \\
\hline FTSS & odds ratio $($ CI 95\%) & odds ratio (CI 95\%) \\
\hline$<8.69$ & Ref. & Ref. \\
$8.69-10.37$ & $0.736(0.293-1.848)$ & $0.743(0.296-1.870)$ \\
$10.38-12.53$ & $0.828(0.336-2.040)$ & $0.856(0.345-2.122)$ \\
$\geq 12.54$ & $3.115(1.390-6.982)$ & $3.337(1.451-7.673)$ \\
\hline 6 MWT & odds ratio $($ CI 95\%) & odds ratio (CI 95\%) \\
\hline$<340.01$ & Ref. & Ref. \\
$340.01-377.80$ & $1.604(0.752-3.422)$ & $1.587(0.739-3.410)$ \\
$377.81-420.00$ & $0.491(0.208-1.156)$ & $0.450(0.187-1.080)$ \\
$\geq 420.01$ & $0.241(0.088-0.662)$ & $0.204(0.070-0.591)$ \\
\hline
\end{tabular}

FTSS: Five timed sit to stand; 6MWT: 6-minnute walk test; CI: confidence interval; Adjust model: sex and age.

\subsection{ROC Curves of 6MWT, TUG Test, and FTSS Test in the Cognitive Frailty Groups}

We observed that an optimal relationship between sensitivity and specificity was achieved at a 6 MWT of $<392.1$, TUG $>8.1$, and FTSS $>12.47$. For this cut-off point, the ROC analysis revealed a sensitivity of $83.61 \%$ and specificity of $47.62 \%$, a sensitivity of $44.26 \%$ and specificity of $79.76 \%$, and a sensitivity of $45.90 \%$ and specificity of $80.95 \%$ for $6 \mathrm{MWT}$, TUG, and FTSS, respectively (Figure 2).
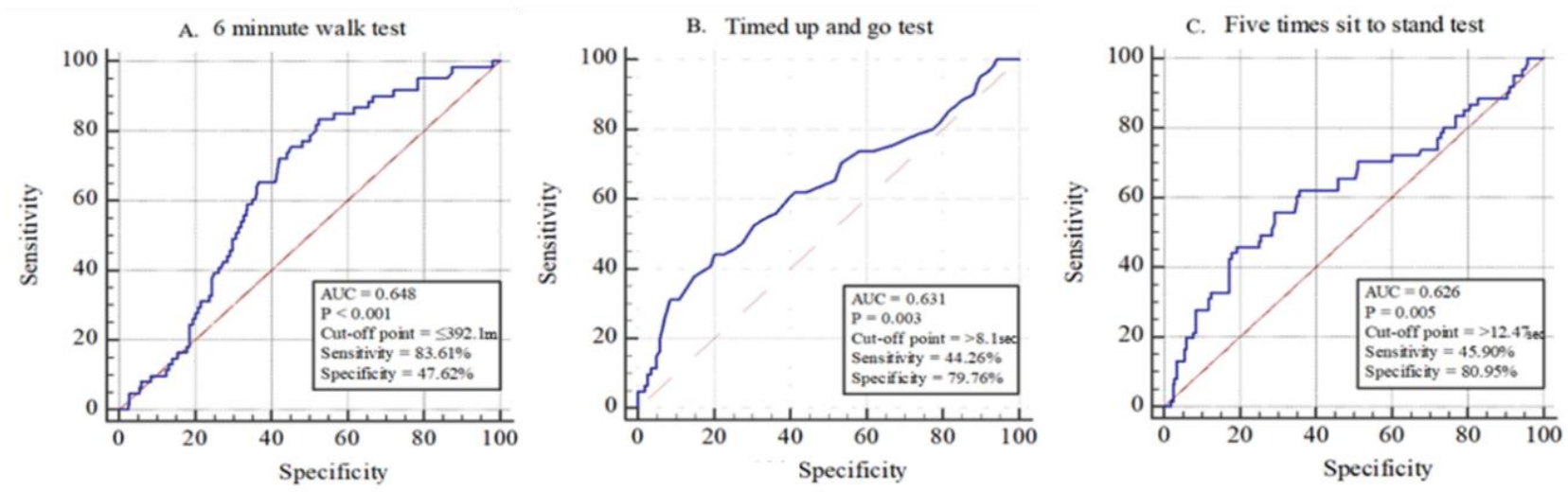

Figure 2. Receiver operating characteristic (ROC) curves of the 6MWT, TUG test, and FTST test in the cognitive frailty groups in relation to the four groups (Blue line: Physical function area; Red line: Reference).

\section{Discussion}

In the present study, cognitive frailty risk was significantly associated with the 6MWT, TUG, and FTSS scores. The participants with cognitive frailty had a lower physical function than those with cognitive decline or physical frailty; however, the association between cognitive frailty and lipid parameters was not observed in this study sample. This finding is inconsistent with the results of previous studies, which demonstrate that, in addition to inflammatory status, some lipid parameters could also be convincingly related to cognitive decline and frailty syndrome [21,22]. With this being said, cognitive frailty prediction is perceived as difficult to identify from simple blood tests. These discrepant results 
were obtained owing to the differences in frailty assessment criteria used to determine frailty status.

Our study supports the new definition of physical function in older adults with cognitive frailty. The AUC (Figure 2) indicated that the 6MWT, TUG, and FTSS tests had the potential to determine the capacity of an older adult in order to predict cognitive frailty; the resulting cutoff points were $\leq 392.1$ for the $6 \mathrm{MWT},>8.1$ for the TUG test, and $>12.47$ for the FTSS test. The 6MWT had a higher sensitivity, while the TUG and FTSS tests had greater specificity. Prior studies on cutoff points and poor performance on the $6 \mathrm{MWT}$ ( $\leq 400 \mathrm{~m}$ ), TUG test ( $\geq 9 \mathrm{~s}$ ), and FTSS test ( $\geq 10 \mathrm{~s}$ ) were useful for stratifying the risk of disability development in community-dwelling older adults [25,27]. Although the previously used cutoff point was different from the one determined by the 6MWT, the difference was not very large [27]. In addition, the FTSS test had a higher cutoff point than the prior study [25]. This result may be due to the fact that the study had more female participants since it is known that women have lesser muscle performance than men [28]. Moreover, this study had a lower cutoff point for TUG than the previous study. The present study had a specificity of $79.56 \%$ for the cutoff point of 8.1 for the TUG test, which is only slightly better than the specificity of the previous study [25]. Additionally, the differences in ethnicity might play a role in the differences in the measurement of the resulting cutoff value $[29,30]$. Therefore, this finding suggests using a cutoff point for cognitive frailty specific to older Korean adults.

We investigated whether the new cutoff point identified in the present study was associated with cognitive frailty in older adults in the community. The results showed significant associations between the TUG test (OR 2.897, 95\% CI 1.283-6.541), FTSS test, (OR 3.337, 95\% CI 1.451-7.673), and 6MWT (OR 0.204, 95\% CI 0.070-0.591) for the cognitive frailty group (Table 3 ). This result was consistent with previous studies showing that the cognitive frailty group had a negative impact on the level of physical function in older adults, which was better than a robust group. Physical frailty includes low physical function, and one's initial cognitive and physical health have been shown to be associated with their subsequent cognitive and physical decline [31]. Therefore, the results showed a higher ratio owing to synergies in physical frailty and cognitive decline. In the present study, an intimate relationship existed between physical function (i.e., low leg strength and low aerobic capacity) and cognitive frailty.

Finally, previous studies suggested that different cognitive frailty groups may be related to an increased risk of functional disability, worsened quality of life, hospitalization, and all-cause mortality. Some large longitudinal population-based studies have shown that different cognitive frailty models may increase the risk of dementia, vascular dementia, and neurocognitive disorders [32]. For different groups of cognitive frailty, physical frailty may precede the onset of cognitive impairment [33]; thus, the present study is useful for older adults for detecting physical frailty.

However, the present study has some limitations. First, a cross-sectional design was used; therefore, the findings do not illustrate any causal relationships between the examined variables. Second, forming generalizations based on this study is difficult as more women were recruited compared to men. Future research should thus consider the ratio of men and women. Third, this study had more individuals under pre-frailty than frailty. Therefore, it must be considered that if there were more participants in the frailty group, the results might have been different.

\section{Conclusions}

In conclusion, our findings indicate that the 6MWT, TUG test, and FTSS test are closely related to cognitive frailty and may be used to determine potential prognostic cutoff points. However, further studies are needed to better define the related cognitive frailty in a multicenter study with a larger sample size. With more research, preventative and new strategies may be developed. 
Author Contributions: Conceptualization: G.-M.K., J.-H.P. and H.P.; methodology: G.-M.K., B.-K.K., Y.L., J.-H.P. and H.P.; investigation: G.-M.K. and D.-R.K.; writing-original draft preparation: G.-M.K., J.-H.P. and H.P.; writing-review and editing: G.-M.K., B.-K.K., J.-H.P. and H.P.; funding acquisition: H.P. All authors have read and agreed to the published version of the manuscript.

Funding: This study was supported by the Korea Health Technology R\&D Project through the Korean Health Industry Development Institute, funded by the Ministry of Health and Welfare, Republic of Korea (grant number: HI15C3207).

Institutional Review Board Statement: The study was conducted according to the guidelines of the Declaration of Helsinki, and approved by the Institutional Review Board of Dong-A university (2015.12).

Informed Consent Statement: Informed consent was obtained from all subjects involved in the study.

Data Availability Statement: Qualified researchers can obtain the data from the corresponding author (htpark@dau.ac.kr). The data are not publicly available due to privacy concerns imposed by the IRB.

Acknowledgments: We are grateful to all study participants and the technical support by the JungGu Health Center, Pusan National University Hospital, and Dong-A University, National Taiwan Normal University research staff.

Conflicts of Interest: The authors declare no conflict of interest.

\section{References}

1. Morley, J.E.; Vellas, B.; Van Kan, G.A.; Anker, S.D.; Bauer, J.M.; Bernabei, R.; Cesari, M.; Chumlea, W.; Doehner, W.; Evans, J.; et al. Frailty Consensus: A Call to Action. J. Am. Med. Dir. Assoc. 2013, 14, 392-397. [CrossRef] [PubMed]

2. Clegg, A.; Barber, S.; Young, J.; Iliffe, S.; Forster, A. The Home-based Older People's Exercise (HOPE) trial: A pilot randomised controlled trial of a home-based exercise intervention for older people with frailty. Age Ageing 2014, 43, 687-695. [CrossRef] [PubMed]

3. Dorner, T.E.; Lackinger, C.; Haider, S.; Luger, E.; Kapan, A.; Luger, M.; Schindler, K.E. Nutritional interven-tion and physical training in malnourished frail community-dwelling elderly persons carried out by trained lay "buddies": Study protocol of a randomized controlled trial. BMC Public Health 2013, 13, 1232. [CrossRef] [PubMed]

4. Tavassoli, N.; Guyonnet, S.; Abellan Van Kan, G.; Sourdet, S.; Krams, T.; Soto, M.E.; Subra, J.; Chicoulaa, B.; Ghisolfi, A.; Balardy, L.; et al. Description of 1,108 older patients referred by their physician to the "Geriatric Frailty Clinic (G.F.C) for assessment of frailty and prevention of disability" at the gerontopole. J. Nutr. Health Aging 2014, 18, 457-464. [CrossRef] [PubMed]

5. Aubertin-Leheudre, M.; Woods, A.J.; Anton, S.; Cohen, R.; Pahor, M. Frailty clinical phenotype: A physical and cognitive point of view. Nestle Nutr. Inst. Workshop Ser. 2015, 83, 55-64. [CrossRef]

6. Kelaiditi, E.; Cesari, M.; Canevelli, M.; Van Kan, G.A.; Ousset, P.-J.; Gillette-Guyonnet, S.; Ritz, P.; Duveau, F.; Soto, M.E.; Provencher, V.; et al. Cognitive frailty: Rational and definition from an (I.A.N.A./I.A.G.G.) International Consensus Group. J. Nutr. Health Aging 2013, 17, 726-734. [CrossRef]

7. Sargent, L.; Brown, R. Assessing the current state of cognitive frailty: Measurement properties. J. Nutr. Health Aging 2016, 21, 152-160. [CrossRef]

8. Ruan, Q.; Yu, Z.; Chen, M.; Bao, Z.; Li, J.; He, W. Cognitive frailty, a novel target for the prevention of elderly dependency. Ageing Res. Rev. 2015, 20, 1-10. [CrossRef]

9. Roppolo, M.; Mulasso, A.; Rabaglietti, E. Cognitive frailty in Italian community-dwelling older adults: Prevalence rate and its association with disability. J. Nutr. Health Aging 2016, 21, 631-636. [CrossRef]

10. Shimada, H.; Makizako, H.; Lee, S.; Doi, T.; Tsutsumimoto, K.; Harada, K.; Hotta, R.; Bae, S.; Nakakubo, S.; Suzuki, T. Impact of cognitive frailty on daily activities in older persons. J. Nutr. Health Aging 2016, 20, 729-735. [CrossRef]

11. Rietman, M.L.; van der A, D.L.; van Oostrom, S.H.; Picavet, H.S.J.; Dollé, M.E.T.; van Steeg, H.; Verschuren, W.M.M.; Spijkerman, A.M.W. The Association between BMI and Different Frailty Domains: A U-Shaped Curve? J. Nutr. Health Aging 2016, 22, 8-15. [CrossRef] [PubMed]

12. Meuleman, J.R.; Brechue, W.F.; Kubilis, P.S.; Lowenthal, D.T. Exercise training in the debilitated aged: Strength and functional outcomes. Arch. Phys. Med. Rehabil. 2000, 81, 312-318. [CrossRef]

13. Aggarwal, N.T.; Wilson, R.S.; Beck, T.L.; Bienias, J.L.; Bennett, D.A. Motor Dysfunction in Mild Cognitive Impairment and the Risk of Incident Alzheimer Disease. Arch. Neurol. 2006, 63, 1763-1769. [CrossRef] [PubMed]

14. Waite, L.; Grayson, D.; Piguet, O.; Creasey, H.; Bennett, H.; Broe, G. Gait slowing as a predictor of incident dementia: 6-year longitudinal data from the Sydney Older Persons Study. J. Neurol. Sci. 2005, 229, 89-93. [CrossRef] [PubMed]

15. Tabue-Teguo, M.; Simo, N.; Harmand, M.G.-C.; Cesari, M.; Avila-Funes, J.A.; Féart, C.; Amiéva, H.; Dartigues, J.-F. Frailty in elderly: A brief review. Gériatrie Psychologie Neuropsychiatrie Viellissement 2017, 15, 127-137. [CrossRef] [PubMed] 
16. Clouston, S.A.; Brewster, P.; Kuh, D.; Richards, M.; Cooper, R.; Hardy, R.; Rubin, M.S.; Hofer, S.M. The dynamic relationship between physical function and cognition in longitudinal aging cohorts. Epidemiol. Rev. 2013, 35, 33-50. [CrossRef] [PubMed]

17. Mossello, E.; Boncinelli, M. Mini-Mental State Examination: A 30-year story. Aging Clin. Exp. Res. 2006, 18, 271-273. [CrossRef]

18. Fried, L.P.; Tangen, C.M.; Walston, J.; Newman, A.B.; Hirsch, C.; Gottdiener, J.; Seeman, T.; Tracy, R.; Kop, W.J.; Burke, G.; et al. Frailty in Older Adults: Evidence for a Phenotype. J. Gerontol. Ser. A Biol. Sci. Med. Sci. 2001, 56, M146-M156. [CrossRef]

19. Pinheiro, P.A.; Passos, T.D.-R.O.; Coqueiro, R.D.S.; Fernandes, M.H.; Barbosa, A.R. [Motor performance of the elderly in northeast Brazil: Differences with age and sex]. Rev. Esc. Enferm. USP 2013, 47, 128-136. [CrossRef]

20. Burr, J.F.; Bredin, S.S.D.; Faktor, M.D.; Warburton, D.E.R. The 6-Minute Walk Test as a Predictor of Objectively Measured Aerobic Fitness in Healthy Working-Aged Adults. Physician Sportsmed. 2011, 39, 133-139. [CrossRef]

21. Morley, J.E. Developing novel therapeutic approaches to frailty. Curr. Pharm. Des. 2009, 15, 3384-3395. [CrossRef] [PubMed]

22. Landi, F.; Russo, A.; Pahor, M.; Capoluongo, E.; Liperoti, R.; Cesari, M.; Bernabei, R.; Onder, G. Serum High-Density Lipoprotein Cholesterol Levels and Mortality in Frail, Community-Living Elderly. Gerontology 2008, 54, 71-78. [CrossRef]

23. Kim, T.H.; Jhoo, J.H.; Park, J.H.; Kim, J.L.; Ryu, S.H.; Moon, S.W.; Choo, I.H.; Lee, D.W.; Yoon, J.C.; Do, Y.J.; et al. Korean Version of Mini Mental Status Examination for Dementia Screening and Its' Short Form. Psychiatry Investig. 2010, 7, 102-108. [CrossRef] [PubMed]

24. Ehrari, H.; Larsen, R.T.; Langberg, H.; Andersen, H.B. Effects of Playful Exercise of Older Adults on Balance and Physical Activity: A Randomized Controlled Trial. J. Popul. Ageing 2020, 13, 207-222. [CrossRef]

25. Makizako, H.; Shimada, H.; Doi, T.; Tsutsumimoto, K.; Nakakubo, S.; Hotta, R.; Suzuki, T. Predictive Cutoff Values of the Five-Times Sit-to-Stand Test and the Timed “Up \& Go" Test for Disability Incidence in Older People Dwelling in the Community. Phys. Ther. 2017, 97, 417-424. [CrossRef]

26. Park, H.; Na, H.R.; Hiroyuki, S.; Kim, W.K.; Jung, M.K. Combined Intervention of Physical Activity, Aerobic Exercise, and Cognitive Exercise Intervention to Prevent Cognitive Decline for Patients with Mild Cognitive Impairment: A Randomized Controlled Clinical Study. J. Clin. Med. 2019, 8, 940. [CrossRef]

27. Kadikar, A.; Maurer, J.; Kesten, S. The six-minute walk test: A guide to assessment for lung transplantation. J. Heart Lung Transplant. 1997, 16, 313-319. [PubMed]

28. Hayashida, I.; Tanimoto, Y.; Takahashi, Y.; Kusabiraki, T.; Tamaki, J. Correlation between Muscle Strength and Muscle Mass, and Their Association with Walking Speed, in Community-Dwelling Elderly Japanese Individuals. PLoS ONE 2014, 9, e111810. [CrossRef]

29. Lear, S.A.; James, P.T.; Ko, G.T.; Kumanyika, S. Appropriateness of waist circumference and waist-to-hip ratio cutoffs for different ethnic groups. Eur. J. Clin. Nutr. 2009, 64, 42-61. [CrossRef]

30. Katzmarzyk, P.T.; Bray, G.A.; Greenway, F.L.; Johnson, W.D.; Newton Jr, R.L.; Ravussin, E.; Ryan, D.H.; Bouchard, C. Ethnicspecific BMI and waist circumference thresholds. Obesity (Silver Spring). 2011, 19, 1272-1278. [CrossRef]

31. Krall, J.R.; Carlson, M.C.; Fried, L.P.; Xue, Q.L. Examining the dynamic, bidirectional associations be-tween cognitive and physical functioning in older adults. Am. J. Epidemiol. 2014, 180, 838-846. [CrossRef] [PubMed]

32. Panza, F.; Lozupone, M.; Solfrizzi, V.; Sardone, R.; Dibello, V.; Di Lena, L.; D’Urso, F.; Stallone, R.; Petruzzi, M.; Giannelli, G. Different cognitive frailty models and health-and cognitive-related outcomes in older age: From epidemiology to prevention. J. Alzheimer's Dis. 2018, 62, 993-1012. [CrossRef] [PubMed]

33. Canevelli, M.; Cesari, M. Cognitive frailty: What is still missing? J. Nutr. Health Aging 2015, 19, 273-275. [CrossRef] [PubMed] 